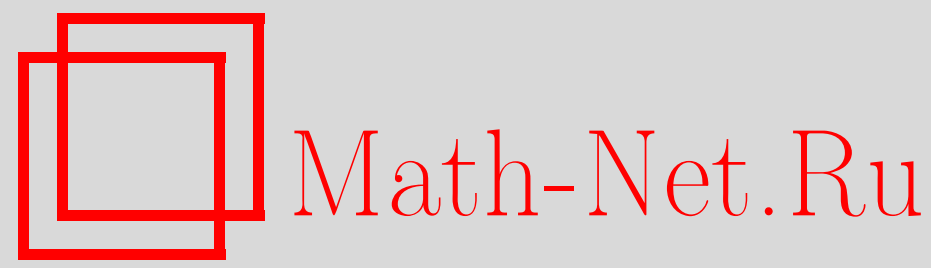

А. М. Стоколос, О скорости сильного дифференцирования интегралов, Матем. заметки, 1996, том 59, выпуск 4, 565-585

DOI: https://doi.org/10.4213/mzm1751

Использование Общероссийского математического портала MathNet.Ru подразумевает, что вы прочитали и согласны с пользовательским соглашением

http://www. mathnet.ru/rus/agreement

Параметры загрузки:

IP : 3.82 .47 .9

26 апреля 2023 г., 15:00:58

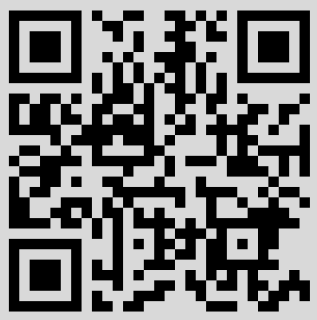




\section{О СКОРОСТИ}

\section{СИЛЬНОГО ДИФФЕРЕНЦИРОВАНИЯ ИНТЕГРАЛОВ}

\section{А. М. Стоколос}

Следствием классической теоремы А. Лебега о дифференцировании интегралов является наличие почти всюду следуюшего фундаментального соотношения

$$
|I|^{-1} \int_{I \ni x}|f(y)-f(x)| d y=o_{x}(1), \quad \operatorname{diam} I \rightarrow 0,
$$

где $I$ обозначает интервалы, a $\operatorname{diam} I$ - их длины. Соотношение (1) выражает некую локальную интегральную непрерывность суммируемых функций. Естественно, что порядок величины $o_{x}(1)$ должен повьшаться с увеличением порядка гладкости функции $f$, и было бы интересньп установить точную зависимость между этими величинами. В терминах интегральной гладкости эта задача получила свое окончательное решение в работе К.И. Осколкова [1]. Для формулировки этого результата нам понадобятся следуюшие объекты.

Пусть $\omega(\delta)$ - модуль непрерывности, не эквивалентный $\delta$. Образуем последовательность $\left\{\delta_{k}\right\}$ по следующему правилу [1]:

$$
\delta_{0}=1, \quad \delta_{k+1}=\min \left\{\delta: \max \left(\frac{\omega(\delta)}{\omega\left(\delta_{k}\right)} ; \frac{\omega\left(\delta_{k}\right) \delta}{\omega(\delta) \delta_{k}}\right) \geqslant \frac{1}{2}\right\},
$$

и определим функцию $\Omega(\delta)=2^{1-k}, \delta_{k+1} \leqslant \delta<\delta_{k}$.

Обозначим также

$$
\begin{aligned}
& D_{\delta}^{*}(f, x)=\inf _{I, x \in I,|I|=\delta}|I|^{-1} \int_{I}|f(y)-f(x)| d y, \\
& D_{\delta}(f, x)=\sup _{I, x \in I,|I|=\delta}|I|^{-1} \int_{I}|f(y)-f(x)| d y .
\end{aligned}
$$

Следуюшая теорема представляет собой существенно ослабленный вариант основного результата из [1]. 
Теорема А. Пусть $\omega(\delta)$ - модуль непрерывности такой, что

$$
\frac{\omega(\delta)}{\delta} \rightarrow \infty, \quad \delta \rightarrow 0
$$

$u \psi(t)$ - невозрастающая положительная функция, для которой

$$
\int_{0}^{1} \frac{d t}{t \psi(t)}<\infty
$$

Тогда для любой функции $f \in H_{1}^{\omega}\left(I^{1}\right)$ почти всюду на $I^{1}$ справедлива оченка

$$
D_{\delta}(f, x)=O_{x}\{\omega(\delta) \psi(\Omega(\delta))\} .
$$

Если же $\psi(t)$ - неотрицательная невозрастающая функиия, для которой

$$
\int_{0}^{1} \frac{d t}{t \psi(t)}=\infty
$$

то существует функиия $f \in H_{1}^{\omega}\left(I^{1}\right)$, для которой почти всюду на $I^{1}$

$$
\varlimsup_{\delta \rightarrow 0} \frac{D_{\delta}^{*}(f, x)}{\omega(\delta) \psi(\Omega(\delta))}=\infty
$$

Распространение результатов К.И. Осколкова на многомерньй случай было произведено В.И. Колядой [2]. Его подход отличается от методов исследований К. И. Осколкова и основан на следующем наблюдении.

Для $f \in L^{1}\left(I^{n}\right)$ и положительной полуаддитивной функции $\varphi(t)$ положим

$$
\mathscr{N}_{\varphi}(f, x)=\sup _{Q, x \in Q}\left(|Q| \varphi\left(|Q|^{1 / n}\right)\right)^{-1} \int_{Q}|f(y)-f(x)| d y,
$$

где $Q$ обозначает куб в $\mathbb{R}^{n}$. Поскольку

$$
|Q|^{-1} \int_{Q \ni x}|f(y)-f(x)| d y \leqslant \mathscr{N}_{\varphi}(f, x) \varphi(\operatorname{diam} Q),
$$

то вопрос о скорости сходимости может быть редуцирован к вопросу о конечности почти всюду $\mathscr{N}_{\varphi}(f, x)$. Соответствующие оценки для $\mathscr{N}_{\varphi}(f, x)$ и были получены В.И. Колядой [2].

А именно, пусть $\omega(\delta)$ - модуль непрерывности. Через $E(\omega)$ обозначим класс всех неотрицательных последовательностей $\left\{\varepsilon_{k}\right\}$ таких, что $(s=1,2, \ldots)$

$$
\sum_{k=0}^{s} 2^{k} \varepsilon_{k} \leqslant 2^{s} \omega\left(2^{-s}\right), \quad \sum_{k=s}^{\infty} \varepsilon_{k} \leqslant \omega\left(2^{-s}\right) .
$$


Тогда условие конечности почти всюду $\mathscr{N}_{\varphi}(f, x)$ для всех $f \in H_{1}^{\omega}\left(I^{n}\right)$ записывается в виде

$$
\sup _{\varepsilon \in E(\omega)} \sum_{k=1}^{\infty} \frac{\varepsilon_{k}}{\varphi\left(2^{-k}\right)}<\infty .
$$

Таким образом, вопрос о скорости дифференширования относительно кубов решен полностью.

Рассмотрим теперь вопрос о скорости сильного дифференцирования. Этот случай сушественно отличается от вьшеизложенного. Дело в том, что соотношение (1), в котором $I=I_{1} \times \cdots \times I_{n}$ - многомерные интервалы, не обязано вьполняться для произвольной суммируемой функции. В силу теоремы Йессена-Марцинкевича-Зигмунда оно справедливо лишь для функции из $L\left(\log ^{+} L\right)^{n-1}\left(I^{n}\right)$ и этот класс не улучшаем (см., например, [3]). Таким образом, прежде чем рассматривать вопросы о скорости, необходимо найти условия на модули, при которых вообще имеет место сходимость. Достаточные условия мгновенно следуют из теорем вложения [4], [5]:

$$
\begin{aligned}
H_{1}^{\omega}\left(I^{n}\right) & \subset L\left(\log ^{+} L\right)^{n-1}\left(I^{n}\right) \Leftrightarrow \int_{0}^{1} \frac{\omega(\delta)}{\delta}\left(\log \frac{1}{\delta}\right)^{n-2} d \delta<\infty, \quad(6) \\
H_{1}^{\omega_{1}, \ldots, \omega_{n}}\left(I^{n}\right) & \subset L\left(\log ^{+} L\right)^{n-1}\left(I^{n}\right) \Leftrightarrow \sum_{i=1}^{n} \int_{0}^{1} \frac{\omega_{i}(\delta)}{\delta}\left(\log \frac{1}{\delta}\right)^{n-2} d \delta<\infty .
\end{aligned}
$$

В [6] было показано, что в изотропном случае условие вложения (6) является также и необходимьм. В анизотропном случае это оказалось не так, что представляется нам весьма интересным моментом.

Во-первых, отметим следуюшее. В условии (7) стоит сумма по всем модулям $\omega_{i}(\delta)$, что является вполне естественным моментом, так как отсутствие контроля гладкости по одному из направлений ведет к отсутствию контроля за глобальным ростом функции в целом, а тогда уже никакого вложения в класс $\Phi(L)$, более узкий, чем $L$, не будет.

С другой стороны, никакой класс $\Phi(L)$, более широкий, чем $L\left(\log ^{+} L\right)^{n-1}\left(I^{n}\right)$, не является хорошим классом с точки зрения сильной дифференцируемости. Тем не менее выяснилось, что в шкале классов $H_{1}^{\omega_{1}, \ldots, \omega_{n}}\left(I^{n}\right)$, гладкость в одном из направлений (естественно считать его "худшим") можно не контролировать вообше.

Во-вторьх, условие на оставшийся $n-1$ модуль непрерьвности является новым по форме. Оно не является точным условием вложения в классы $\Phi(L)$ в $(n-1)$-мерном случае. Это условие записьвается следуюшим образом.

Пусть $\theta_{k}^{i}$ такие, что

$$
\omega_{i}\left(\theta_{k}^{i}\right)=2^{-k}, \quad i=1, \ldots, n, \quad k=0, \ldots, \infty .
$$


Тогда сходимость ряда

$$
\sum_{k=0}^{\infty} 2^{-k} \prod_{i=1}^{n-1} \log \frac{1}{\theta_{k}^{i}}<\infty
$$

является достаточньп для сильного дифференцирования интегралов функций из $H_{1}^{\omega_{1}, \ldots, \omega_{n}}\left(I^{n}\right)$. На классе регулярных модулей непрерывностей это условие окончательно (детали см. в [6]).

Теперь уже можно непосредственно переходить к вопросу о скорости сходимости почти всюду.

Классический подход к изучению вопросов сильного дифференцирования, предложенньй впервые, по всей видимости, А. Зигмундом, состоит в мажорировании многомерного оператора композицией частных одномерных операторов. Тогда вопросы конечности почти всюду сводятся к вопросам суммируемости и принадлежности некоторьм классам Орлича соответствуюших частных операторов, т.е. к некоторьм теоремам вложения. Для максимальной функции Харди-Литлвуда эти результаты хорошо известны (см., например, [3]).

Предложенной схемой можно воспользоваться и в нашем случае. Двумерный результат не требует предварительной подготовки и на нем можно сразу же продемонстрировать высказанный тезис.

Пусть $f \in H_{1}^{\omega}\left(I^{2}\right), x \in I=I_{1} \times I_{2}$. Тогда

$$
\begin{aligned}
|I|^{-1} \int_{I} \mid f(y)- & f(x) \mid d y \\
\leqslant & \left|I_{1}\right|^{-1} \int_{I_{1}}\left|I_{2}\right|^{-1} \int_{I_{2}}\left|f\left(y_{1}, y_{2}\right)-f\left(y_{1}, x_{2}\right)\right| d y_{1} d y_{2} \\
& \quad+\left|I_{1}\right|^{-1} \int_{I_{1}}\left|f\left(x_{1}, x_{2}\right)-f\left(y_{1}, x_{2}\right)\right| d y_{1} \\
\leqslant & \left(\mathscr{M}^{1} \circ \mathscr{N}_{\psi}^{2} f\right)(x) \psi\left(\left|I_{2}\right|\right)+\left(\mathscr{N}_{\varphi}^{1} f\right)(x) \varphi\left(\left|I_{1}\right|\right) .
\end{aligned}
$$

Здесь $\mathscr{M}^{1}$ обозначает максимальную функцию Харди-Литлвуда по первой переменной, $\mathscr{N}_{\varphi}^{1}$ обозначает $\mathscr{N}_{\varphi}$-функцию по первой переменной, а $\mathscr{N}_{\psi}^{2}$ обозначает $\mathscr{N}_{\varphi}$-функцию по второй переменной. Конечность почти всюду $\mathscr{N}_{\varphi}^{1}$ обсуждалась выше, а конечность почти всюду $\mathscr{M}^{1} \circ \mathscr{N}_{\psi}^{2}$ сводится к вопросу о суммируемости $\mathscr{N}_{\psi}^{2}$, решение которого содержится в [2]. А именно, условие

$$
\int_{0}^{1} \frac{\omega(t)}{t \psi(t)} d t<\infty
$$

является необходимым и достаточным для суммируемости $\mathscr{N}_{\varphi} f$ для всех функций $f \in H_{1}^{\omega}\left(I^{n}\right)$. 
Таким образом, если $\varphi(t)$ удовлетворяет $(5)$, а $\psi(t)$ удовлетворяет (9), то для любой функции $f \in H_{1}^{\omega}\left(I^{2}\right)$ почти всюду на $I^{2}$ справедлива оценка

$$
|I|^{-1} \int_{I \ni x}|f(y)-f(x)| d y=O_{x}\left\{\varphi\left(\left|I_{1}\right|\right)+\psi\left(\left|I_{2}\right|\right)\right\} .
$$

Соотношение (10) является количественным уточнением (1) в случае, когда $I$ - двумерньй интервал.

Естественно, что правая часть (10) может быть заменена более точными, но и более сложными выражениями. Например, в силу инвариантности изотропных классов Гёльдера относительно выбора нумерации осей координат, справедлива аналогичная оценка с заменой $I_{1}$ и $I_{2}$ местами. Если обозначить $\Omega_{i j}(I)=\max \left(\varphi\left(\left|I_{i}\right|\right) ; \psi\left(\left|I_{j}\right|\right)\right)$, а

$$
\Omega=\min \left\{\Omega_{1,2}(I) ; \Omega_{2,1}(I)\right\},
$$

то (10) можно переписать в виде

$$
|I|^{-1} \int_{I \ni x}|f(y)-f(x)| d y=O_{x}\{\Omega(I)\} .
$$

Здесь уже характеристика $\Omega(I)$ зависит не только от абсолютньх размеров $I$, но и от величины "вытянутости". Безусловно, подбор адекватных характеристик в правой части (10) является важной задачей, требующей дальнейших разработок. Однако, как нам кажется, важно не упустить достаточную прозрачность терминов, в которых выражается связь между размерами интервала и величиной малости $o_{x}$ в (1). Этого принципа мы старались придерживаться в настоящей работе и в изотропном случае. Как нам кажется, это удалось.

Теорема 1. Пусть $n \geqslant 2, \omega(\delta)$ - модуль непрерывности, для котоporo

$$
\int_{0}^{1} \frac{\omega(\delta)}{\delta}\left(\log \frac{1}{\delta}\right)^{n-2} d \delta<\infty
$$

Пусть $\psi(\delta)$ - положительная неубьвающая функиия, для которой

$$
\int_{0}^{1} \frac{\omega(\delta)}{\delta \psi(\delta)}\left(\log \frac{1}{\delta}\right)^{n-2} d \delta<\infty
$$

Тогда для любой функиии $f \in H_{1}^{\omega}\left(I^{n}\right)$ почти всюду на $I^{n}$ справедлива оценка

$$
|I|^{-1} \int_{I \ni x}|f(y)-f(x)| d y=O_{x}\{\psi(\operatorname{diam} I)\}, \quad I=I_{1} \times \cdots \times I_{n} .
$$


Анизотропньй же случай традиционно гораздо более сложен для изучения, по крайней мере в вопросах, связанных с интегральной гладкостью. При этом естественно требовать, чтобы в случае одинаковых мажорант частных модулей непрерьвностей анизотропные характеристики преврашались в изотропные.

Как это часто бывает в теоремах вложения, поиск вида соответствуюших условий представляет, пожалуй, решающую трудность, которую необходимо сначала преодолеть. В связи с этим введем в рассмотрение следуюшие характеристики.

Считая, для простоты, $\omega_{i}(t)$ возрастаюшими функциями с условиями нормировки $\omega_{i}(1)=1$, определим функции $\phi_{i j}(t)$ следуюшим образом:

$$
\omega_{i}\left(\phi_{i j}(t)\right)=\omega_{j}(t), \quad i, j=1, \ldots, n, \quad t \in(0,1) .
$$

Положим также

$$
\begin{gathered}
A(j)=\{k: 1 \leqslant k \leqslant n, k \neq j\}, \quad A(j, s)=\{k \in A(j): k \neq s\}, \\
w_{i}(t)=\prod_{j \in A(i, n)} \log \frac{1}{\phi_{j i}(t)} .
\end{gathered}
$$

Теперь заметим, что в изотропном случае, очевидно, $\phi_{i j}(t)=t$, поэтому

$$
w_{i}(t)=\prod_{j \in A(i, n)} \log \frac{1}{t}=\left(\log \frac{1}{t}\right)^{n-2} .
$$

Это и есть согласование анизотропного и изотропного случаев. Вместо (11) появляется условие

$$
\sum_{i=1}^{n-1} \int_{0}^{1} \frac{\omega_{i}(t) w_{i}(t)}{t} d t<\infty
$$

В [6] условие (13) присутствует при доказательстве основного результата. Именно оно является достаточным условием для сильного дифференцирования интеграла функции из $H_{1}^{\omega_{1}, \ldots, \omega_{n}}\left(I^{n}\right)$. Условие (8) влечет (13), но является, с нашей точки зрения, более удобным и прозрачным, тем более, что для регулярньх модулей (8) окончательно.

Что касается условия на $n$-й модуль непрерывности, то оно записывается в виде

$$
\sum_{k=0}^{\infty} \frac{\omega_{n}\left(\delta_{k}\right)}{\psi_{n}\left(\delta_{k}\right)}<\infty
$$

где $\left\{\delta_{k}\right\}$ - разбиваюшая последовательность, построенная для $\omega_{n}(\delta)$. Это условие, на самом деле, - другая форма записи условия К.И. Осколкова (см. теорему А). В таком виде оно применялось в диссертации А.А. Соляника [7].

Теперь мы готовы сформулировать основную теорему данной работы в анизотропном случае. 
ТЕОРема 2. Пусть $\omega_{i}(\delta)$ - модули непрерывности такие, что

$$
\sum_{i=1}^{n-1} \int_{0}^{1} \frac{\omega_{i}(t) w_{i}(t)}{t} d t<\infty
$$

$u\left\{\delta_{k}\right\}-$ разбивающая последовательность, построенная для $\omega_{n}(\delta)$. Пусть $\psi_{j}(t)$ - положительные неубывающие функиии, для которых

$$
\sum_{k=0}^{\infty} \frac{\omega_{n}\left(\delta_{k}\right)}{\psi_{n}\left(\delta_{k}\right)}+\sum_{i=1}^{n-1} \int_{0}^{1} \frac{\omega_{i}(t) w_{i}(t)}{t \psi_{i}(t)} d t<\infty
$$

Тогда для любой функиии $f \in H_{1}^{\omega_{1}, \ldots, \omega_{n}}\left(I^{n}\right)$ для почти всех $x \in I^{n}$ справедлива оченка

$$
|I|^{-1} \int_{I \ni x}|f(y)-f(x)| d y=O_{x}\left\{\max _{1 \leqslant i \leqslant n} \psi_{i}\left(\left|I_{i}\right|\right)\right\}, \quad I=I_{1} \times \cdots \times I_{n} .
$$

Доказательство этой теоремы в целом следует обозначенной схеме, т.е. вводится некоторьй "сильный” аналог $\mathscr{N}_{\varphi}$-функции, исследуется вопрос о его суммируемости, а затем колебание оценивается через композиции частных максимальных функций Харди-Литлвуда и сильных $\mathscr{N}_{\varphi}$-функций всевозможных размерностей, меньших $n$.

Несмотря на прозрачность идейных соображений, их техническая реализация, учитьвая разветвленность $n$-мерной структуры, является весьма нетривиальным моментом. Здесь очень удобньм инструментом оказались введенные функции $\phi_{i j}(t)$. Точнее, их хорошие групповые свойства, выраженные в следуюшей лемме, в которой $\varphi_{i j}(t)$ обозначает обратную к $\phi_{i j}(t)$ функцию.

Лемма А. Для любого $t \in(0,1) u$ любых $i, j=1, \ldots, n, k=0, \ldots, \infty$ справедливы следующие равенства:

$$
\phi_{i j}\left(\phi_{j k}(t)\right)=\phi_{i k}(t) ; \quad \varphi_{i j}(t)=\phi_{j i}(t) ; \quad \phi_{i i}(t)=t ; \quad \phi_{i j}\left(\theta_{k}^{j}\right)=\theta_{k}^{i} .
$$

Кроме этой леммы нам понадобятся и другие.

Лемма 1. Пусть $f \in L\left(I^{n}\right), f(x)=0$ для $x \notin I^{n}$. Тогда

$$
\int_{\mathbb{R}^{n}}\left|\Delta_{h}^{i} f(x)\right| d x \leqslant C(f) \omega_{i}(f, h), \quad i=1, \ldots, n .
$$

В этой лемме левая часть отличается от правой на интегралы от функции $f$ по множествам меры $h$, возникающих при сдвиге. Очевидно, они мажорируются (с некоторой константой) суммой $\omega_{i}(f, h)+h\|f\|$.

Следующая лемма очевидна. 
Лемма 2. Пусть $g(t)>0$ такова, что

$$
\int_{0}^{1} \frac{g(t)}{t} d t<\infty
$$

Тогда

$$
\sum_{k=0}^{\infty} 2^{k} \int_{0}^{2^{-k}} g(t) d t \leqslant 2 \int_{0}^{1} \frac{g(t)}{t} d t .
$$

Теперь мы переходим к лемме, которая вбирает в себя основную трудность работы с многомерными подобластями в $I^{n}$. Именно здесь и реализуется упоминавшаяся выше арифметика функций $\phi_{i j}(t)$.

А именно, пусть теперь $\tau \equiv(\tau(1), \ldots, \tau(k))$ - некоторая перестановка набора $(1, \ldots, k)$. Обозначим

$$
\alpha_{s} \equiv \phi_{\tau(s), \tau(s-1)}\left(x_{\tau(s-1)}\right)
$$

и в этих терминах определим криволинейные симплексы в $I^{n}$ :

$E_{\tau} \equiv\left\{\left(x_{1}, \ldots, x_{n}\right): 0 \leqslant x_{\tau(1)} \leqslant 1, \alpha_{2} \leqslant x_{\tau(2)} \leqslant 1, \ldots, \alpha_{n} \leqslant x_{\tau(n)} \leqslant 1\right\}$.

Рассмотрим пример. Пусть $n=2, \omega_{1}(t)=t, \omega_{2}(t)=\sqrt{t}$. В этом случае возможны лишь две перестановки набора $(1,2)$ : тождественная, т.е. $\tau:(1,2) \rightarrow(1,2)$, и циклическая, т.е. $\tau:(1,2) \rightarrow(2,1)$.

Первому случаю соответствует

$$
E_{1,2}=\left\{\left(x_{1}, x_{2}\right): 0 \leqslant x_{\tau(1)} \leqslant 1, \alpha_{2} \leqslant x_{\tau(2)} \leqslant 1\right\} .
$$

Здесь $\alpha_{2}=\phi_{\tau(2), \tau(1)}\left(x_{\tau(1)}\right)=\phi_{2,1}\left(x_{1}\right)=x_{1}^{2}$, так как подстановка $\phi_{2,1}(t)$ переводит $\omega_{2}(t)=\sqrt{t}$ в $\omega_{1}(t)=t$. Итак,

$$
E_{1,2}=\left\{\left(x_{1}, x_{2}\right): 0 \leqslant x_{1} \leqslant 1, x_{1}^{2} \leqslant x_{2} \leqslant 1\right\} .
$$

Во втором случае $\alpha_{2}=\phi_{1,2}\left(x_{2}\right)=\sqrt{x_{2}}$, так как подстановка $\phi_{1,2}(t)$ переводит $\omega_{1}(t)=t$ в $\omega_{2}(t)=\sqrt{t}$. Тогда

$$
E_{2,1}=\left\{\left(x_{1}, x_{2}\right): 0 \leqslant x_{2} \leqslant 1, \sqrt{x_{2}} \leqslant x_{1} \leqslant 1\right\} .
$$

Идея, лежащая в основе доказательства леммы 3 , состоит в замене порядка следования координат в определении множества $E$, поскольку (см. рис. 1)

$$
E_{2,1}=\left\{\left(x_{1}, x_{2}\right): 0 \leqslant x_{1} \leqslant 1,0 \leqslant x_{2} \leqslant x_{1}^{2}\right\}
$$

и, таким образом, исключая саму параболу, $\chi_{E_{2,1}}+\chi_{E_{1,2}}=1$ на $I^{2}$. 


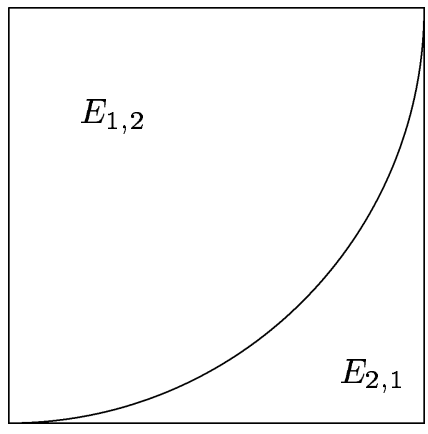

Рис. 1

ЛЕмма 3. Для всех $x \in I^{n}$

$$
1 \leqslant \sum_{\tau} \chi_{\tau}(x) \leqslant n !
$$

әде сумма берется по всевозмозиным перестановкам набора $\tau$, a $\chi_{\tau}$ обозначает характеристическую функиию мнохества $E_{\tau}$.

ДокАЗАТЕЛьСтво. Правое неравенство очевидно. Докажем левое. Очевидно,

$$
\begin{aligned}
1=\int_{I^{n}} d x_{1} \cdots d x_{n}= & \int_{I^{n-2}} d x_{1} \cdots d x_{n-2} \int_{0}^{1} d x_{n-1} \int_{\phi_{n, n-1}\left(x_{n-1}\right)}^{1} d x_{n} \\
& +\int_{I^{n-2}} d x_{1} \cdots d x_{n-2} \int_{0}^{1} d x_{n-1} \int_{0}^{\phi_{n, n-1}\left(x_{n-1}\right)} d x_{n} .
\end{aligned}
$$

Во втором интеграле поменяем местами порядок интегрирования по $x_{n-1}$ и $x_{n}$ :

$$
\int_{0}^{1} d x_{n-1} \int_{0}^{\phi_{n, n-1}\left(x_{n-1}\right)} d x_{n}=\int_{0}^{1} d x_{n} \int_{\varphi_{n, n-1}\left(x_{n}\right)}^{1} d x_{n-1}
$$

Учитьвая, что $\varphi_{i j}(t)=\phi_{j i}(t)$, получим

$$
\begin{aligned}
1= & \int_{0}^{1} d x_{1} \cdots \int_{0}^{1} d x_{n-2} \int_{0}^{1} d x_{n-1} \int_{\phi_{n, n-1}\left(x_{n-1}\right)}^{1} d x_{n} \\
& +\int_{0}^{1} d x_{1} \cdots \int_{0}^{1} d x_{n-2} \int_{0}^{1} d x_{n} \int_{\phi_{n-1, n}\left(x_{n}\right)}^{1} d x_{n-1} .
\end{aligned}
$$

Применив к каждому из двух получившихся слагаемых описанную процедуру для внешнего $(n-1)$-мерного интеграла, получим четыре слагаемых, 
к которым снова применим описанную процедуру. Продолжая по индукции, получим

$$
1=\sum_{\tau \in T} \int_{0}^{1} d x_{\tau(1)} \int_{\alpha_{2}}^{1} d x_{\tau(2)} \cdots \int_{\alpha_{n-1}} d x_{\tau(n)}=\int_{I^{n}} \sum_{\tau \in T} \chi_{\tau}(x) d x
$$

где сумма берется по множеству индексов, содержащему $2^{n}$ элементов.

Поскольку, по построению, интегралы в предыдущем равенстве получались последовательным разбиением области интегрирования, то подинтегральная сумма не превосходит почти всюду единицы, и, таким образом, лемма 3 доказана.

С помощью этой леммы мы можем заменить интегрирование по $I^{n}$ интегрированием по множествам $E_{\tau}$, в которых последние $n-1$ переменных интегрирования отделены от нуля.

Теперь введем сильные $\mathscr{N}_{\varphi}$-функции, полагая

$$
\mathcal{N}_{\varphi}^{s}(f, x)=\sup _{I, x \in I}\left(|I| \max _{1 \leqslant i \leqslant n} \varphi_{i}\left(\left|I_{i}\right|\right)\right)^{-1} \int_{I}|f(y)-f(x)| d y
$$

а также соответствуюшие весовые функции

$$
W_{i}(t)=\prod_{j \in A(i)} \log \frac{1}{\phi_{j i}(t)}
$$

ТЕОРема 3. Пусть $\omega_{i}(\delta)$ - модули непрерывности такие, что

$$
\sum_{i=1}^{n} \int_{0}^{1} \frac{\omega_{i}(t) W_{i}(t)}{t} d t<\infty
$$

а $\psi_{j}(t)$ - неубывающие положсительные функиии, для которых

$$
\sum_{i=1}^{n} \int_{0}^{1} \frac{\omega_{i}(t) W_{i}(t)}{t \psi_{i}(t)} d t<\infty
$$

Тогда для любой функиии $f \in H_{1}^{\omega_{1}, \ldots, \omega_{n}}\left(I^{n}\right)$

$$
\mathcal{N}_{\psi}^{s}(f, x) \in L^{1}\left(I^{n}\right)
$$


ДоКАЗАТЕЛЬСТВо. Введем следуюшие обозначения. Пусть $I=I_{1} \times$ $\cdots \times I_{n}$, знак « обозначает неравенство, справедливое с некоторой константой, множества $A(j)$ и $A(j, s)$ определены вьше. Через

$$
I(j)=\prod_{k \in A(j)} I_{k}, \quad I(j, s)=\prod_{k \in A(j, s)} I_{k}
$$

обозначим подинтервалы $I$, а через

$$
d y(j)=\prod_{k \in A(j)} d y_{k}, \quad d y(j, s)=\prod_{k \in A(j, s)} d y_{k}
$$

обозначим дифференциалы соответствуюших размерностей.

Далее, пусть $m_{j}-$ натуральные числа, для которых

$$
2^{-m_{j}-1}<\left|I_{j}\right| \leqslant 2^{-m_{j}} .
$$

Положим теперь $v(I)=\left(2^{-m_{1}}, \ldots, 2^{-m_{n}}\right)$.

В силу леммы 3 для $x$ и $y$ из $I^{n}$ имеем

$$
\begin{aligned}
|f(y)-f(x)| \leqslant & \sum_{\tau}\left|f\left(y_{1}, \ldots, x_{\tau(1)}, \ldots, y_{n}\right)-f\left(y_{1}, \ldots, y_{n}\right)\right| \chi_{\tau}(v(I)) \\
& +\sum_{\tau}\left|f\left(y_{1}, \ldots, x_{\tau(1)}, \ldots, y_{n}\right)-f\left(x_{1}, \ldots, x_{n}\right)\right| \chi_{\tau}(v(I)),
\end{aligned}
$$

откуда

$$
\begin{aligned}
& |I|^{-1} \int_{I}|f(y)-f(x)| d y \\
& \quad \leqslant \sum_{\tau}|I|^{-1} \int_{I}\left|f\left(y_{1}, \ldots, y_{n}\right)-f\left(y_{1}, \ldots, x_{\tau(1)}, \ldots, y_{n}\right)\right| d y \cdot \chi_{\tau}(v(I)) \\
& \quad+n ! \sum_{j=1}^{n}|I(j)|^{-1} \int_{I(j)}\left|f\left(x_{1}, \ldots, x_{n}\right)-f\left(y_{1}, \ldots, x_{j}, \ldots, y_{n}\right)\right| d y(j) .
\end{aligned}
$$

Пусть

$$
\begin{aligned}
\mathscr{R}_{\psi}^{\tau}(f, x)= & \sup _{I, x \in I, v(I) \in E_{\tau}}\left(|I| \psi_{\tau(1)}\left(\left|I_{\tau(1)}\right|\right)\right)^{-1} \\
& \times \int_{I}\left|f\left(y_{1}, \ldots, y_{n}\right)-f\left(y_{1}, \ldots, x_{\tau(1)}, \ldots, y_{n}\right)\right| d y, \\
\mathscr{N}_{\psi}^{s, j}(f, x)= & \sup _{I, x \in I}\left(|I(j)| \max _{i \in A(j)} \psi_{i}\left(\left|I_{i}\right|\right)\right)^{-1} \\
& \times \int_{I(j)}\left|f\left(x_{1}, \ldots, x_{n}\right)-f\left(y_{1}, \ldots, x_{j}, \ldots, y_{n}\right)\right| d y(j) .
\end{aligned}
$$


Так как для любого $i=1, \ldots, n$

$$
\frac{1}{\max _{1 \leqslant j \leqslant n} \psi_{j}\left(\left|I_{j}\right|\right)} \leqslant \frac{1}{\psi_{i}\left(\left|I_{i}\right|\right)}
$$

TO

$$
\mathscr{N}_{\psi}^{s}(f, x) \leqslant \sum_{\tau} \mathscr{R}_{\psi}^{\tau}(f, x)+n ! \sum_{j=1}^{n} \mathscr{N}_{\psi}^{s, j}(f, x) .
$$

Поскольку во второй сумме фактически стоят $(n-1)$-мерные $\mathscr{N}_{\varphi}^{s}$-функции, то, с учетом итеративных соображений, достаточно показать суммируемость $\mathscr{R}_{\psi}^{\tau}(f, x)$.

Обозначим

$$
E_{\tau}^{\star}=\left\{\left(m_{1}, \ldots, m_{n}\right):\left(2^{-m_{1}}, \ldots, 2^{-m_{n}}\right) \in E_{\tau}\right\} .
$$

Заменяя супремум по $I$ супремумом по наборам $\left(2^{-m_{1}}, \ldots, 2^{-m_{n}}\right) \in E_{\tau}$, а затем и на сумму по этим наборам, получим

$$
\begin{aligned}
\mathscr{R}_{\psi}^{\tau}(f, x) \ll & \sum_{\left(m_{1}, \ldots, m_{n}\right) \in E_{\tau}^{\star}} 2^{m_{1}} \int_{-2^{-m_{1}}}^{2^{-m_{1}}} d t_{1} \ldots \\
& \times 2^{m_{n}} \int_{-2^{-m_{n}}}^{2^{-m_{n}}} \mid f\left(x_{1}+t_{1}, \ldots, x_{n}+t_{n}\right) \\
& \quad-f\left(x_{1}+t_{1}, \ldots, x_{\tau(1)}, \ldots, x_{n}+t_{n}\right) \mid d t_{n} \\
& \times\left[\psi_{\tau(1)}\left(2^{\left.-m_{\tau(1)}\right)}\right]^{-1}\right. \\
\ll & \quad \sum_{\left(m_{1}, \ldots, m_{n}\right) \in E_{\tau}^{\star}\left|\varepsilon_{1}\right|=1, \ldots,\left|\varepsilon_{n}\right|=1} 2^{m_{1}} \int_{0}^{2^{-m_{1}}} d t_{1} \ldots \\
& \times 2^{m_{n}} \int_{0}^{2^{-m_{n}}} \mid f\left(x_{1}+\varepsilon_{1} t_{1}, \ldots, x_{n}+\varepsilon_{n} t_{n}\right) \\
& \quad-f\left(x_{1}+\varepsilon_{1} t_{1}, \ldots, x_{\tau(1)}, \ldots, x_{n}+\varepsilon_{n} t_{n}\right) \mid d t_{n} \\
& \times\left[\psi_{\tau(1)}\left(2^{\left.-m_{\tau(1)}\right)}\right]^{-1} .\right.
\end{aligned}
$$

Отсюда

$$
\begin{aligned}
\left\|\mathscr{R}_{\psi}^{\tau}(f, x)\right\|_{1} \ll & \sum_{\left|\varepsilon_{1}\right|=1, \ldots,\left|\varepsilon_{n}\right|=1} \sum_{\left(m_{1}, \ldots, m_{n}\right) \in E_{\tau}^{\star}} 2^{m_{\tau(1)}} \\
& \times\left[\psi_{\tau(1)}\left(2^{\left.-m_{\tau(1)}\right)}\right]^{-1} \int_{0}^{2^{-m_{\tau(1)}}}\left\|\Delta_{\varepsilon_{\tau(1)} t}^{\tau(1)} f(x)\right\|_{1} d t .\right.
\end{aligned}
$$

Оценим произвольное слагаемое. Не ограничивая обшности, считаем

$$
\varepsilon_{1}=\cdots=\varepsilon_{n}=1, \quad \tau(1)=1, \ldots, \tau(n)=n .
$$


Тогда

$$
\begin{aligned}
& \sum_{\left(m_{1}, \ldots, m_{n}\right) \in E_{\tau}^{\star}} 2^{m_{1}}\left[\psi_{1}\left(2^{-m_{1}}\right)\right]^{-1} \int_{0}^{2^{-m_{1}}}\left\|\Delta_{t}^{1} f(x)\right\|_{1} d t \\
& \ll \sum_{m_{1}=0}^{\infty}\left\{\sum_{m_{2} \in M_{2}} \ldots \sum_{m_{n} \in M_{n}} 1\right\} 2^{m_{1}}\left[\psi_{1}\left(2^{-m_{1}}\right)\right]^{-1} \int_{0}^{2^{-m_{1}}}\left\|\Delta_{t}^{1} f(x)\right\|_{1} d t,
\end{aligned}
$$

где $M_{k}=\left\{m_{k}: \phi_{k, k-1}\left(2^{-m_{k-1}}\right) \leqslant 2^{-m_{k}}\right\}$. Сумму в фигурных скобках подсчитаем по индукции. Имеем

$$
\begin{aligned}
\sum_{m_{n-1} \in M_{n-1}} \sum_{m_{n} \in M_{n}} 1 & \ll \sum_{m_{n-1} \in M_{n-1}} \log \frac{1}{\phi_{n, n-1}\left(2^{\left.-m_{n-1}\right)}\right.} \\
& \ll \log \frac{1}{\phi_{n, n-1}\left(\phi_{n-1, n-2}\left(2^{\left.-m_{n-2}\right)}\right)\right.} \sum_{m_{n-1} \in M_{n-1}} 1 .
\end{aligned}
$$

Однако, в силу леммы $3 \phi_{n, n-1}\left(\phi_{n-1, n-2}\left(2^{-m_{k-2}}\right)\right)=\phi_{n, n-2}\left(2^{-m_{k-2}}\right)$. Таким образом,

$$
\sum_{m_{n-1} \in M_{n-1}} \sum_{m_{n} \in M_{n}} 1 \ll \log \frac{1}{\phi_{n, n-2}\left(2^{\left.-m_{n-2}\right)}\right.} \log \frac{1}{\phi_{n-1, n-2}\left(2^{\left.-m_{n-2}\right)}\right.} .
$$

Продолжая эту процедуру по индукции, получим

$$
\sum_{m_{2} \in M_{2}} \cdots \sum_{m_{n} \in M_{n}} 1 \ll \prod_{j=2}^{n} \log \frac{1}{\phi_{j, 1}\left(2^{\left.-m_{1}\right)}\right.} .
$$

Теперь, учитывая монотонность функций $\psi_{1}(t)$ и $\phi_{j, 1}(t)$, будем иметь

$$
\begin{aligned}
\left\|\mathscr{R}_{\psi}^{\tau}(f, x)\right\|_{1} & \ll \sum_{\left(m_{1}, \ldots, m_{n}\right) \in E_{\tau}^{\star}} 2^{m_{1}}\left[\psi_{1}\left(2^{-m_{1}}\right)\right]^{-1} \int_{0}^{2^{-m_{1}}}\left\|\Delta_{t}^{1} f(x)\right\|_{1} d t \\
& \ll \sum_{m_{1}=0}^{\infty} \prod_{j=2}^{n} \log \frac{1}{\phi_{j, 1}\left(2^{-m_{1}}\right)} \cdot 2^{m_{1}} \int_{0}^{2^{-m_{1}}} \frac{\left\|\Delta_{t}^{1} f(x)\right\|_{1}}{\psi_{1}(t)} d t \\
& \ll \sum_{m_{1}=0}^{\infty} 2^{m_{1}} \int_{0}^{2^{-m_{1}}} \frac{\left\|\Delta_{t}^{1} f(x)\right\|_{1}}{\psi_{1}(t)} \prod_{j=2}^{n} \log \frac{1}{\phi_{j, 1}(t)} d t .
\end{aligned}
$$

Применение лемм 1 и 2 дает

$$
\left\|\mathscr{R}_{\psi}^{\tau}(f, x)\right\|_{1} \ll \int_{0}^{1} \frac{\omega_{1}(t)}{t \psi_{1}(t)} \prod_{j=2}^{n} \log \frac{1}{\phi_{j, 1}(t)} d t=\int_{0}^{1} \frac{\omega_{1}(t) W_{1}(t)}{t \psi_{1}(t)} d t<\infty .
$$

Теорема 3 доказана. 
СлЕДСТВИЕ 1. Пусть $\omega(\delta)$ - модуль непрерывности, для которого

$$
\int_{0}^{1} \frac{\omega(\delta)}{\delta}\left(\log \frac{1}{\delta}\right)^{n-1} d \delta<\infty,
$$

а $\psi(t)$ - положительная возрастающая функиия, для которой

$$
\int_{0}^{1} \frac{\omega(\delta)}{\delta \psi(\delta)}\left(\log \frac{1}{\delta}\right)^{n-1} d \delta<\infty .
$$

Тогда для любой функиии $f \in H_{1}^{\omega}\left(I^{n}\right)$

$$
\mathcal{N}_{\psi}^{s}(f, x) \in L^{1}\left(I^{n}\right) .
$$

ДоКАЗАТЕЛЬСТВо. Как и ранее, достаточно заметить, что в изотропном случае $\phi_{j i}(t)=t$.

Перейдем теперь к доказательству теоремы 2 . Пусть $f \in H_{1}^{\omega_{1}, \ldots, \omega_{n}}\left(I^{n}\right)$ и $\widehat{x}$ означает отсутствие соответствующей координаты в векторной записи, например $\widehat{x}_{1}=\left(x_{2}, \ldots, x_{n}\right)$. Тогда

$$
\begin{aligned}
|I|^{-1} \int_{I \ni x} \mid f(y) & -f(x) \mid d y \\
\leqslant & \left|I_{n}\right|^{-1} \int_{I_{n}}|I(n)|^{-1} \int_{I(n)}\left|f\left(y_{1}, \widehat{y}_{1}\right)-f\left(y_{1}, \widehat{x}_{1}\right)\right| d y_{1} d \widehat{y}_{1} \\
& +\left|I_{n}\right|^{-1} \int_{I_{n}}\left|f\left(x_{1}, \widehat{x}_{1}\right)-f\left(y_{1}, \widehat{x}_{1}\right)\right| d y_{1} \\
\leqslant & \left(\mathscr{M}^{1} \circ \mathscr{N}_{\psi}^{s, n}\right)(f, x)\left\{\max _{1 \leqslant j \leqslant n} \psi_{j}\left(\left|I_{j}\right|\right)\right\}+\mathscr{N}_{\psi_{n}}^{1}(f, x) \psi_{n}\left(\left|I_{n}\right|\right) .
\end{aligned}
$$

Конечность почти всюду первого слагаемого следует из суммируемости $\mathscr{N}_{\psi}^{s, n}(f, x)$, которая, с учетом размерности, обеспечивается сходимостью интегралов в (14).

Конечность почти всюду второго слагаемого следует из сходимости ряда в (14). Действительно, если

$$
\int_{0}^{1} \frac{d t}{t \widetilde{\psi}(t)}<\infty
$$

или, что тоже самое,

$$
\sum_{k=1}^{\infty} \frac{1}{\widetilde{\psi}\left(2^{-k}\right)}<\infty
$$

то в силу теоремы А

$$
|I|^{-1} \int_{I \ni x}|f(y)-f(x)| d y \leqslant C(x)\{\omega(|I|) \widetilde{\psi}(\Omega(|I|))\} .
$$


С другой стороны,

$$
\sum_{k=1}^{\infty} \frac{1}{\widetilde{\psi}\left(2^{-k}\right)}=\sum_{k=1}^{\infty} \frac{\omega\left(\delta_{k}\right)}{\omega\left(\delta_{k}\right) \widetilde{\psi}\left(\Omega\left(\delta_{k}\right)\right)} .
$$

Обозначив $\psi(t)=\omega(t) \widetilde{\psi}(\Omega(t))$, получим $\mathscr{N}_{\psi}(f, x)<C(x)$ при условии

$$
\sum_{k=0}^{\infty} \frac{\omega\left(\delta_{k}\right)}{\psi\left(\delta_{k}\right)}<\infty
$$

Аналогичным образом доказьвается конечность почти всюду $\mathscr{N}_{\psi_{n}}^{1}(f, x)$. Таким образом, теорема 2 доказана.

Теорема 1 является очевидным следствием теоремы 2 в силу согласования изотропного и анизотропного случаев.

Обсудим теперь точность оценки из теоремы 1 . Ограничимся двумерным случаем, но прежде с делаем следующее одномерное наблюдение.

Во-первых, $\Omega(t) \gg t$ и, таким образом, для $f \in H_{1}^{\omega}\left(I^{1}\right)$ почти всюду на $I^{1}$ справедлива оценка

$$
|I|^{-1} \int_{I \ni x}|f(y)-f(x)| d y=O_{x}\{\omega(|I|) \psi(|I|)\}, \quad \operatorname{diam} I \rightarrow 0, \quad x \in I .
$$

Во-вторьх, если модуль непрерывности удовлетворяет условию БариСтечкина

$$
\int_{0}^{\delta} \frac{\omega(t)}{t} d t+\delta \int_{\delta}^{1} \frac{\omega(t)}{t^{2}} d t=O(\omega(\delta)),
$$

то для разбивающей последовательности $\left\{\delta_{k}\right\}$ справедливо соотношение

$$
\frac{\delta_{k}}{\delta_{k+1}}=O(1)
$$

Отсюда следует, что с некоторым $p \in(0 ; 1)$

$$
\delta \leqslant \Omega(\delta) \leqslant C \delta^{p} .
$$

Пусть теперь $\psi(t)$ удовлетворяет соотношению

$$
\int_{0}^{1} \frac{d t}{t \psi(t)}=\infty
$$

Определим функцию $\sigma(t)=\psi\left(t^{1 / p} / C\right)$. Тогда

$$
\int_{0}^{1} \frac{d t}{t \sigma(t)}=\infty
$$


и в силу теоремы А для некоторой функщии $f \in H_{1}^{\omega}\left(I^{1}\right)$

$$
\varlimsup_{\delta \rightarrow 0} \frac{D_{\delta}^{*}(f, x)}{\omega(\delta) \sigma(\Omega(\delta))}=\infty .
$$

Однако, $\sigma(\Omega(\delta)) \geqslant \sigma\left(C \delta^{p}\right)=\psi(\delta)$, и, следовательно, почти всюду

$$
\varlimsup_{\delta \rightarrow 0} \frac{D_{\delta}^{*}(f, x)}{\omega(\delta) \psi(\delta)}=\infty .
$$

Таким образом, из теоремы А можно извлечь следующее следствие.

СЛЕДСТВИЕ А. Пусть $\omega(\delta)$ - модуль непрерывности, не әквивалентный $\delta$. Если $\psi(t) \in \Phi-$ полоэсительная неубываюшая функиия, для которой

$$
\int_{0}^{1} \frac{\omega(t)}{t \psi(t)}<\infty,
$$

то для любой функиии $f \in H_{1}^{\omega}\left(I^{1}\right)$ почти всюду на $I^{1}$ справедлива ои,енка

$$
D_{\delta}(f, x)=O_{x}\{\psi(\delta)\} .
$$

Если жее $\omega(\delta)$ - модуль непрерывности, удовлетворяющий условию Бари-Стечкина, а для функиии $\psi(t)$ условиие (15) не выполнено, то существует функиия $f \in H_{1}^{\omega}\left(I^{1}\right)$, для которой

$$
\varlimsup_{\delta \rightarrow 0} \frac{D_{\delta}^{*}(f, x)}{\psi(\delta)}=\infty .
$$

Таким образом, для модулей непрерьвности, удовлетворяющих условию Бари-Стечкина, точность оценки, полученной в теореме 1 , следует из следствия А.

Условие Бари-Стечкина означает степенной рост модуля, однако, не наилучший. Оно нарушается при подходе как к быстро растушим модулям типа $\omega(\delta)=\delta$ (за счет второго слагаемого), так и при подходе к медленно растушим модулям, типа $\omega(\delta)=\{\log (1 / \delta)\}^{-1}$ (за счет первого слагаемого).

Для быстро растущих модулей одномерные контрпримеры оставляют зазор между оценками снизу и сверху порядка логарифмического множителя. Вопрос о том, как его убрать, остается открытьм. С нашей точки зрения он достаточно принципиальный, так как в одномерном случае при подходек $\omega(\delta)=\delta$ качество сходимости улучшается, а для $\omega(\delta)=\delta$ и вовсе не происходит потерь (по сравнению со сходимостью по норме). Будет ли улучшаться качество сходимости в двумерном случае - нам не известно.

С другой стороны, для медленно растуших модулей одномерные контрпримеры не подходят совсем, так как при подходе модулей к $\omega(\delta)=\{\log (1 / \delta)\}^{-1}$ сходимость ухудшается, а потом исчезает и вовсе. 
В полном соответствии с этим ухудшается и качество оценки в теореме 1 ввиду все более и более медленной сходимости интеграла в (12). Однако, нам удалось показать, что в этом случае оценка остается точной для регулярньх модулей непрерьвности. Ей даже удается придать вид оценки из следствия А.

С этой целью обозначим

$$
D_{\delta}^{s}(f, x)=\sup _{\substack{I, x \in I \\ \operatorname{diam} I=\delta}}|I|^{-1} \int_{I}|f(y)-f(x)| d y .
$$

Тогда, учитьвая положительньй результат для изотропного случая, приведем следуюшую теорему.

Теорема 4. (i) Пусть $\omega(\delta)$ - модуль непрерывности, не эквивалентный $\delta$, такой, что

$$
\int_{0}^{1} \frac{\omega(t)}{t} d t<\infty
$$

$u \psi(t)$ - полохительная неубывающая функиия, удовлетворяющая условию (15). Тогда для любой функиии $f \in H_{1}^{\omega}\left(I^{2}\right)$ почти всюду на $I^{2}$ справедлива оченка

$$
D_{\delta}^{s}(f, x)=O_{x}\{\psi(\delta)\} .
$$

(ii) Если же (15) не выполняется, а модуль непрерывности удовлетворяет условию невозрастания функиии $\omega(t) t^{-1 / 2}, u \omega(t) / \psi(t)$ не убъвает, то существует функиия $f \in H_{1}^{\omega}\left(I^{2}\right)$, для которой

$$
\varlimsup_{\delta \rightarrow 0} \frac{D_{\delta}^{s}(f, x)}{\psi(\delta)}=\infty,
$$

почти всюду на $I^{2}$.

ДокАЗАТЕЛЬСтво. Оценка сверху следует из теоремы 1. Докажем оценку снизу. Легко проверить, что условие регулярности модуля непрервности относительно $\sqrt{t}$ обеспечивает вьполнение следующего равенства: $\omega\left(\delta_{k}\right)=2^{-k}$. Тогда, учитьвая монотонность отношения $\omega(t) / \psi(t)$, получим

$$
\int_{0}^{1} \frac{\omega(\delta)}{\delta \psi(\delta)} d \delta \leqslant \sum_{k=1}^{\infty} \int_{\delta_{k}}^{\delta_{k-1}} \frac{\omega(\delta)}{\delta \psi(\delta)} d \delta \leqslant \sum_{k=1}^{\infty} \frac{\omega\left(\delta_{k-1}\right)}{\psi\left(\delta_{k-1}\right)} \log \frac{\delta_{k-1}}{\delta_{k}} .
$$

Таким образом,

$$
\sum_{k=1}^{\infty} \frac{\omega\left(\delta_{k-1}\right)}{\psi\left(\delta_{k-1}\right)} \log \frac{\delta_{k-1}}{\delta_{k}}=\infty
$$


Теперь возможны две ситуации. Первая, когда расходится ряд

$$
\sum_{k=1}^{\infty} \frac{\omega\left(\delta_{k}\right)}{\psi\left(\delta_{k}\right)}=\infty
$$

В этом случае легко видеть, что требуемая оценка следует из одномерного случая, так как сходимость интеграла

$$
\int_{0}^{1} \frac{1}{\delta \psi_{0}(\delta)} d \delta
$$

эквивалетна сходимости ряда

$$
\sum_{k=1}^{\infty} \frac{1}{\psi_{0}\left(2^{-k}\right)}=\sum_{k=1}^{\infty} \frac{1}{\psi_{0}\left(\Omega\left(\delta_{k}\right)\right)} .
$$

При этом с некоторой функцией $f \in H_{1}^{\omega}\left(I^{1}\right)$ справедливо соотношение

$$
\varlimsup_{\delta \rightarrow 0} \frac{D_{\delta}^{*}(f, x)}{\omega(\delta) \psi_{0}(\Omega(\delta))}=\infty
$$

Если теперь $\psi_{0}(\Omega(t)) \omega(t)=\psi(t)$, то последнее соотношение перепишется в виде

$$
\varlimsup_{\delta \rightarrow 0} \frac{D_{\delta}^{*}(f, x)}{\psi(\delta)}=\infty .
$$

При этом оно имеет место почти всюду при наличии (16).

Таким образом, остался второй случай, когда

$$
\sum_{k=1}^{\infty} \frac{\omega\left(\delta_{k}\right)}{\psi\left(\delta_{k}\right)}<\infty
$$

Введем множества

$$
Q_{k}=\left[0, \delta_{k}\right]^{2}, \quad E_{k}=\left\{\left(x_{1}, x_{2}\right): \delta_{k} \leqslant x_{1} \leqslant \delta_{k-1}, 0 \leqslant x_{2} x_{1} \leqslant \delta_{k} \delta_{k-1}\right\} .
$$

Тогда

$$
\left|E_{k}\right|=\delta_{k} \delta_{k-1} \log \frac{\delta_{k-1}}{\delta_{k}}, \quad m_{k}=\left[\frac{\omega\left(\delta_{k-1}\right)}{\psi\left(\delta_{k-1}\right) \delta_{k-1} \delta_{k}}\right]+1
$$

и, таким образом,

$$
m_{k}\left|E_{k}\right| \geqslant \frac{\omega\left(\delta_{k-1}\right)}{\psi\left(\delta_{k-1}\right)} \log \frac{\delta_{k-1}}{\delta_{k}}
$$

Следовательно,

$$
\sum_{k=1}^{\infty} m_{k}\left|E_{k}\right|=\infty
$$


Тогда по лемме Кальдерона о сдвигах (см., например, [3]) определим сдвиги $\tau_{i}^{k}, i=1, \ldots, m_{k}, k \geqslant 0$, так, чтобы верхний предел множеств $\tau_{i}^{k}\left(E_{k}\right)$ имел полную меру. Положим

$$
\lambda_{k}=\psi\left(\delta_{k-1}\right) \frac{\delta_{k-1}}{\delta_{k}}
$$

и получим

$$
\lambda_{k} m_{k} \delta_{k}^{2} \leqslant 2 \omega\left(\delta_{k-1}\right)=4 \omega\left(\delta_{k}\right) .
$$

Обозначив теперь через $\chi_{i}^{k}$ характеристическую функцию множества $\tau_{i}^{k}\left(E_{k}\right)$, определим функции

$$
f_{k}^{i}=\lambda_{k} \chi_{i}^{k}, \quad f=\sum_{k=1}^{\infty} \sum_{i=1}^{m_{k}} f_{k}^{i} .
$$

Тогда для $\delta_{j-1}<h \leqslant \delta_{j}$ и для $i=1,2$, с учетом свойств $\left\{\delta_{k}\right\}$ (см. [1]), имеем

$$
\begin{aligned}
\omega_{i}(f, h) & \ll h \sum_{k=1}^{j} \frac{\lambda_{k} m_{k} \delta_{k}^{2}}{\delta_{k}}+\sum_{k=j+1}^{\infty} \lambda_{k} m_{k} \delta_{k}{ }^{2} \\
& \ll h \sum_{k=1}^{j} \frac{\omega\left(\delta_{k}\right)}{\delta_{k}}+\sum_{k=j+1}^{\infty} \omega\left(\delta_{k}\right) \ll \omega(h) .
\end{aligned}
$$

Таким образом, $f \in H_{1}^{\omega}\left(I^{2}\right)$.

Далее, с учетом (17),

$$
\sum_{k=1}^{\infty} m_{k}\left|Q_{k}\right| \ll \sum_{k=1}^{\infty} \frac{\omega\left(\delta_{k-1}\right)}{\psi\left(\delta_{k-1}\right)} \cdot \frac{\delta_{k}}{\delta_{k-1}} \ll \sum_{k=1}^{\infty} \frac{\omega\left(\delta_{k}\right)}{\psi\left(\delta_{k}\right)}<\infty
$$

Следовательно, мера верхнего предела множеств $\tau_{i}^{k}\left(Q_{k}\right)$ равна нулю, и, таким образом, почти каждая точка $x$ содержится в конечном числе носителей функций $f_{k}^{i}$.

Пусть теперь $F=\lambda_{k} \chi_{Q_{k}}$. Тогда для $x \in E_{k}$ можно подобрать $I \ni x$ (см. рис. 2) такой, что

$$
\begin{aligned}
|I|^{-1} \int_{I}|F(y)-F(x)| d y & =|I|^{-1} \int_{I}|F(y)| d y=\frac{\lambda_{k}\left|Q_{k}\right|}{|I|} \\
& \geqslant \frac{\lambda_{k} \delta_{k}^{2}}{\delta_{k} \delta_{k-1}}=\psi\left(\delta_{k-1}\right) .
\end{aligned}
$$




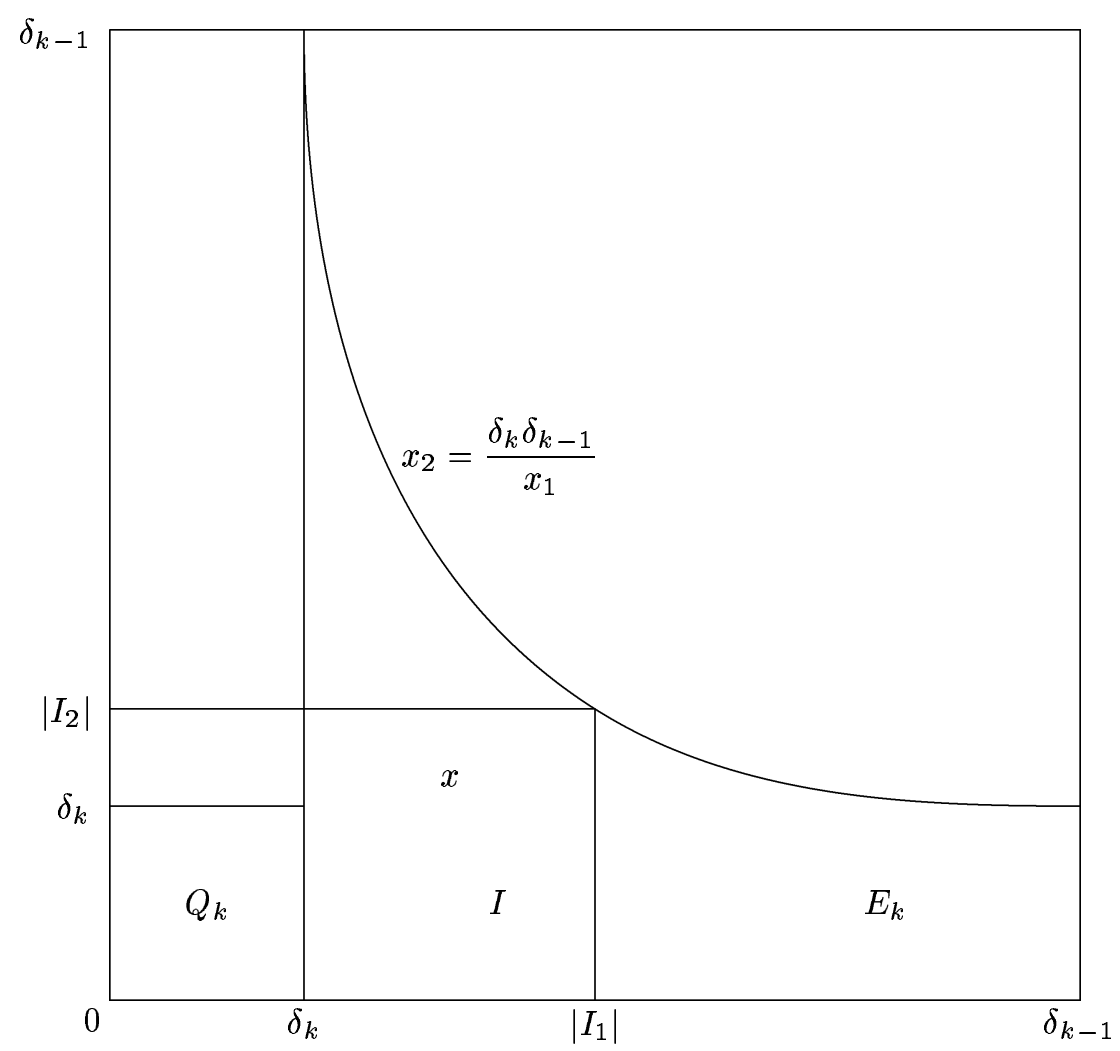

Рис. 2

Обозначив через $l(I)=\max \left(\left|I_{1}\right| ;\left|I_{2}\right|\right)$, получим

$$
|I|^{-1} \int_{I}|F(y)-F(x)| d y \geqslant \psi(l(I)) .
$$

Обозначим $Q_{i}^{k}=\tau_{i}^{k}\left(Q_{k}\right)$. Пусть теперь $x$ принадлежит не более, чем $n$ кубам $Q_{i}^{k}$. Пусть $x \in I$ и $\operatorname{diam} I$ настолько мал, чтобы либо $I$ входил целиком в $Q_{i}^{k}$, либо не пересекался с $Q_{i}^{k}$ для $k \leqslant n$, а для $k=n$ выполняется соотношение типа (18). Тогда

$$
\begin{aligned}
|I|^{-1} \int_{I}|f(y)-f(x)| d y \geqslant & \left.\left|\sum_{k=1}^{n-1} \sum_{i=1}^{m_{k}}\right| I\right|^{-1} \int_{I}\left[f_{k}^{i}(y)-f_{k}^{i}(x)\right] d y \\
& +\sum_{i=1}^{m_{n}}|I|^{-1} \int_{I}\left[f_{n}^{i}(y)-f_{n}^{i}(x)\right] d y \\
& +\sum_{k=n+1}^{\infty} \sum_{i=1}^{m_{k}}|I|^{-1} \int_{I}\left[f_{k}^{i}(y)-f_{k}^{i}(x)\right] d y \mid \\
= & \left|\Sigma_{1}+\Sigma_{2}+\Sigma_{3}\right| .
\end{aligned}
$$


В силу взаимного расположения $I$ и носителей $f_{k}^{i} \quad \Sigma_{1}=0$. Далее, с учетом (18), $\Sigma_{2} \geqslant \psi(l(I))$, а так как $\Sigma_{3} \geqslant 0$, то $\Sigma_{1}+\Sigma_{2}+\Sigma_{3} \geqslant \psi(l(I))$, откуда и следует требуемое. Теорема 4 доказана.

СледСтвиЕ 2. (i) Пусть $\omega(\delta)=\delta^{\alpha}, 0<\alpha<1$. Тогда для любой функиии $f \in H_{1}^{\omega}\left(I^{2}\right)$ для почти всех $x \in I^{2}$

$$
|I|^{-1} \int_{I \ni x}|f(y)-f(x)| d y=O_{x}\left\{(\operatorname{diam} I)^{\alpha-\varepsilon}\right\}
$$

где $\varepsilon>0$. Положить $\varepsilon=0$ нельзя.

(ii) Пусть $\omega(\delta)=\{\log (1 / \delta)\}^{-\alpha}, \alpha>1$. Тогда для любой функиии $f \in H_{1}^{\omega}\left(I^{2}\right)$ для почти всех $x \in I^{2}$

$$
|I|^{-1} \int_{I \ni x}|f(y)-f(x)| d y=O_{x}\{\log (1 / \operatorname{diam} I)\}^{-\alpha+\varepsilon},
$$

әде $\varepsilon>0$. Положить $\varepsilon=0$ нельзя.

В заключение автор выражает благодарность Ю. В. Крякину за полезные обсуждения и помошь при подготовке работы к публикации.

Одесский государственный университет

Поступило

Институт математики, экономики и механики

13.01 .95

E-mail : root@opi.odessa.ua

\section{СПИСОК ЦИТИРОВАННОЙ ЛИТЕРАТУРЫ}

[1] Осколков К.И. Аппроксимативные свойства суммируемых функций на множествах полной меры // Матем. сб. 1977. Т. 103 (145). С. 563-589.

[2] Коляда В.И. Оценки максимальных функций, связанных с локальной гладкостью // Докл. АН СССР. 1987. Т. 293. №3.

[3] Гусман М. Дифференцирование интегралов в $\mathbb{R}^{n}$. М.: Мир, 1978.

[4] Ульянов П. Л. Вложение некоторых классов функций $H_{p}^{\omega} / /$ Изв. АН СССР. 1968. T. 32. C. $649-686$.

[5] Панджикидзе Л. К. Теоремы вложения для функций многих переменных // Сообщ. АН ГССР. 1970. Т. 60. № 1. С. 29-31.

[6] Стоколос А.М. О сиљном дифференцировании интегралов функций из классов Гёльдера // Матем. заметки. 1994. Т. 55. №1. С. 84-104.

[7] Соляник А.А. О приближении функций из $H^{p}$ в верхней полуплоскости. Дисс. ... к. ф.-м.н.. Одесса: ОГУ, 1985. 